\title{
Electromagnetic Radiation from a Source in a Plasma*
}

\author{
G. W. ForD \\ Department of Physies, Lniversity of Michigan, Ann Arbor, Michigan and Space Technology \\ Labaratories, Los Angeles, California
}

\begin{abstract}
The problem of determining the electromagnetic radiations from a given charge-current distribution situated in a plasma in the presence of a uniform external magnetic field is solved. Explicit expressions for the asymptotic electromagnetic fields are given for the case of a cold plasma. The resulting expressions simplify considerably in the case where the frequency of the wave is much less than the electron gyrof requency or the plasma frequency. Here it is found that the radiation is confined to a cone whose generator makes a constant angle of about $20^{\circ}$ with the external magnetic field. The fields diverge on this cone but the divergence is so weak as to cause no physical difficulty. Another result of interest is that a purely longitudinal current, of which the simplest example is a pulsating spherically symmetric charge distribution, will radiate in this medium while, as is well known, such a charge distribution will not radiate in an isotropic medium. As an example, the low-frequency spectrum of radiation from a radial burst of charge is calculated.
\end{abstract}

\section{INTRODUCTION}

This paper is devoted to the problem of determining the radiation field arising from a given charge-current distribution which is immersed in a plasma in the presence of a uniform external magnetic field. 'This problem is of special interest, aside from practical considerations, because the external magnetic field provides a screw axis and hence the medium is anisotropic, or more precisely, gyrotropic. This anisotropy is extremely large at frequencies below the gyrofrequency of the plasma electrons in the extemal magnetic field. Indeed, it is difficult to imagine another medium in which the anisotropy is so large. One of the striking effects to which this anisotropy leads is the confinement of the low frequency radiation field within a cone whose vertex is at the source and whose generator makes a constant angle of about $20^{\circ}$ with the external magnetic field. We find, in fact, that the fields diverge on this cone but that the divergence is so weak as to cause

* Supported in part by the Office of Naval Research. Part of this work was done in the summer of 1960 when the author was visiting the Space Technology Laboratories, Los Angeles, California.

${ }^{1}$ A general introduction to the electromagnetic properties of such a medium is given by Ratcliffe (1). 
110 physical difficulty. ${ }^{2}$ Another effect of some importance is the fact that a purely longitudinal current distribution, of which the simplest example is a radially pulsating sphere of charge, will radiate in this medium, while, as is well known, such a distribution will not radiate in an isotropic medium. An obvious application of this cffect is the ealculation of the very low-frequency radio waves emitted by a nuclear explosion in the upper atmosphere, for which we would predict intense radiation, while the same blast occurring in the lower regions of the atmosphere would radiate weakly if at all.

In Section II we show how the Maxwell equations in this medium can be reduced to a single (rather complicated) partial differential equation for the transverse part of the dielectric displacement field. Then in Section III this equation is solved for the asymptotic fields far from the source of radiation. Since the radiated electromagnetic energy and its angular distribution can be determined from the asymptotic fields alone this amounts to a complete solution of the problem. The resulting expressions, however, are rather complicated because of the romplicated form of the indices of refraction and of the dielectric relation. In order to make these expressions more amenable to discussion, in Section IV we discuss the results in the low frequency limit. Finally, in Section $V$ the application of these results to the problem of determining the low-frequency radiation from a radial burst of fast electrons is discussed.

\section{ELIMINATION OF THE LONGITUDINAL FIELD}

For a specified charge density $\rho(\mathbf{r}, \mathbf{t})$ and current density $\mathbf{j}(\mathbf{r}, t)$ the Maxwell equations for the electromagnetic fields are:

$$
\begin{aligned}
\operatorname{div} \mathbf{B} & =0 \\
\operatorname{curl} \mathbf{E}+(1 / c)(\partial \mathbf{B} / \partial t) & =0 \\
\operatorname{div} \mathbf{D} & =4 \pi \rho \\
\operatorname{curl} \mathbf{B}-(1 / c)(\partial \mathbf{D} / \partial t) & =(4 \pi / c) \mathbf{j} .
\end{aligned}
$$

Here we have used the fact that the magnetic field $\mathbf{H}$ is equal to the magnetic induction $\mathbf{B}$ for a plasma. The relation between the dielectric displacement $\mathbf{D}$ and the electric field $\mathbf{E}$ takes its simplest form if we introduce the time Fourier transforms of these quantities. In general we shall adopt the notation that the time Fourier transforms of a function $f(\mathbf{r}, t)$ is $f_{\omega}(\mathbf{r})$ with

$$
f_{\omega}(\mathbf{r})=\frac{1}{2 \pi} \int_{-\infty}^{\infty} d t e^{i \omega t} f(\mathbf{r}, t), \quad f(\mathbf{r}, t)=\int_{-\infty}^{\infty} d \omega e^{-i \omega t} f_{\omega}(\mathbf{r})
$$

${ }^{2}$ In this respect the behavior of the fields is similar to that in the Cerenkov effect. See, e.g., Jelley (2). 
If we assume the temperature of the plasma is low enough that the mean thermal velocity of the plasma electrons is small compared with the phase velocity of the electromagnetic waves, (i.e., the index of refraction $n \ll \sqrt{m c^{2} k T}$ ), then the relation between $D_{\omega}$ and $E_{\omega}$ is given by":

$$
\mathbf{E}_{\omega}=\eta_{1} \mathbf{D}_{\omega}+\left(\eta_{3}-\eta_{1}\right) \hat{b} \cdot \mathbf{D}_{\omega} \hat{b}+i \eta_{2} \hat{b} \times \mathbf{D}_{\omega} .
$$

In this expression $\hat{b}$ is a unit vector whose direction is that of the external magnetic field, and $\eta_{1}, \eta_{2}$, and $\eta_{\text {: }}$ are functions of the frequency $\omega$. If the motion of the heavy ions is neglected these functions may be expressed in terms of two complex parameters:

$$
x=\omega_{p}^{2} / \omega(\omega+i \nu), \quad y=\omega_{i} / \omega+i \nu,
$$

where $\nu$ is the electron collision frequency in the plasma, $\omega_{0}=e B / m c$, is the clectron gyrofrequency with $B$ the magnitude of the external magnetic field, $e$ and $m$ the electron charge and mass, and $c$ the velocity of light in a vacuum, and where $\omega_{p}=\left(4 \pi N e^{2} / m\right)^{1 / 2}$ is the plasma frequency with $N$ the number density of electrons in a plasma. In terms of $x$ and $y$ the functions $\eta_{1}, \eta_{2}$, and $\eta_{3}$ in $(2.3)$ are given by:

$$
\eta_{1}=\frac{1-x-y^{2}}{(1-x)^{2}-y^{2}}, \quad \eta_{2}=\frac{x y}{(1-x)^{2}-y^{2}}, \quad \eta_{: 3}=\frac{1}{1-x} .
$$

If we take the Fourier transform of the Maxwell equations above we get

$$
\begin{aligned}
\operatorname{div} \mathbf{B}_{\omega} & =0, \\
\operatorname{curl} \mathbf{E}_{\omega}-i(\omega / c) \mathbf{B}_{\omega} & =0, \\
\operatorname{div} \mathbf{D}_{\omega} & =4 \pi \rho_{\omega} \\
\operatorname{curl} \mathbf{B}_{\omega}+i(\omega / c) \mathbf{D}_{\omega} & =(4 \pi / c) \mathbf{j}_{\omega}
\end{aligned}
$$

Except for the special case $\omega=0$, which will not be important in our discussion, the first Maxwell equation is now a consequence of the second which may be written:

$$
\mathrm{B}_{\omega}(\mathrm{r})=-i(c / \omega) \operatorname{curl} \mathrm{E}_{\omega} .
$$

Csing this expression for $\mathrm{B}_{\omega}$, the fourth Maxwell equation becomes:

$$
\text { r'url curl } \mathbf{E}_{\omega}-\left(\omega^{2} / c^{2}\right) \mathbf{D}_{\omega}=i\left(4 \pi \omega / c^{2}\right) \mathbf{j}_{\omega} \text {. }
$$

The third Maxwell equation is seen to be a consecpuenee of this equation if we rocognize that

${ }^{3}$ This equation can also be written $E=\varepsilon^{-1} D$, where $\varepsilon^{-1}$ is the (matrix) inverse of the dielectric tensor $\varepsilon$. An expression for $\varepsilon$ is given at the bottom of p. 184 in Ref 2 . The effects arising when the plasma is not considered "cold" are discussed by burnel.t (3). 


$$
\operatorname{div} \mathbf{j}_{\omega}=i \omega \rho_{\omega}
$$

which is just the time Fourier transform of the continuity equalion. Hence (2.8) together with (2.3) represents an equation for $\mathbf{D}_{\omega}$. Once we have an expression for $\mathbf{D}_{\omega}$ we obtain $\mathrm{E}_{\omega}$ from $(2.3)$ and $\mathbf{B}_{\omega}$ from (2.7).

As a first step in solving for $\mathbf{D}_{\omega}$ we recall the theorem that any vector field can be uniquely separated into a longitudinal part, with vanishing curl, and a transverse part, with vanishing divergence. ${ }^{4}$ Hence we can write

$$
\mathbf{D}_{\omega}(\mathbf{r})=\mathbf{D}_{\omega}^{\prime}(\mathbf{r})+\mathbf{D}_{\omega}^{\prime \prime}(\mathbf{r})
$$

where

$$
\operatorname{div} \mathbf{D}_{\omega}^{\prime}=0, \quad \text { curl } \mathbf{D}_{\omega}^{\prime \prime}=0 .
$$

Inserting (2.10) in (2.8) gives:

$$
\begin{aligned}
\operatorname{curl} \text { curl } \mathbf{E}_{\omega}{ }^{\prime}-\left(\omega^{2} / c^{2}\right) & \mathbf{D}_{\omega}{ }^{\prime} \\
& =i\left(4 \pi \omega / c^{2}\right) \mathbf{j}_{\omega}-\operatorname{curl} \operatorname{curl} \mathbf{E}_{\omega}{ }^{\prime \prime}+\left(\omega^{2} / c^{2}\right) \mathbf{D}_{\omega}{ }^{\prime \prime} .
\end{aligned}
$$

In this expression we mean by $\mathbf{E}_{\omega}{ }^{\prime}$ and $\mathbf{E}_{\omega}{ }^{\prime \prime}$ not the longitudinal and transverse parts of $\mathbf{E}_{\omega}$, but rather the expressions resulting when the longitudinal and transverse parts of $D_{\omega}$ are inserted in (2.3). The right-hand side of (2.12) will be known if $\mathbf{D}_{\omega}{ }^{\prime \prime}$ is known, and we can obtain an equation for $\mathbf{D}_{\omega}$ " by taking the divergence of both sides of $(2.12)$. The result is the equation:

$$
\operatorname{div} \mathrm{D}_{\omega}^{\prime \prime}=4 \pi \rho_{\omega},
$$

where we have used (2.9). Equation (2.13) is the well-known Poisson equation, whose solution is ${ }^{5}$ :

$$
\mathbf{D}_{\omega}^{\prime \prime}(\mathbf{r})=-\operatorname{grad} \int d \mathbf{r}^{\prime} \frac{\rho_{\omega}\left(\mathbf{r}^{\prime}\right)}{\mid \mathbf{r}-\overline{\mathbf{r}^{\prime} \mid}} .
$$

Taking the inverse Fourier transform of this result we find

$$
\mathrm{D}^{\prime \prime}(\mathbf{r}, t)=-\operatorname{grad} \int d \mathbf{r}^{\prime} \frac{\rho\left(\mathbf{r}^{\prime}, t\right)}{\left|\mathbf{r}-\mathbf{r}^{\prime}\right|} .
$$

This is clearly just the instantaneous Coulomb field arising from the charge distribution $\rho(\mathbf{r}, t)$. If we assume the charge distribution is confined within a region of dimension $a$, then, fixing the origin of our coordinate system somewhere near the charge distribution, we find for $r \gg a$,

$$
\mathbf{D}^{\prime \prime}(\mathbf{r}, t) \sim Q \mathbf{r} / r^{3}
$$

${ }^{4}$ See, e.g., Panofsky and Phillips (4). This separation, which is sometimes called the separation into irrotational and solenoidal parts, is just the separation into longitudinal and transverse components of the spatial Fourier transform of the field.

${ }^{5}$ See, e.g., Stratton (5); also Ref. 4, p. 9. 
where

$$
Q=\int d \mathbf{r} \rho(\mathbf{r}, t)
$$

is the lotal charge in the charge distribution. ${ }^{6}$ Hence, we see that $\mathbf{D}^{\prime \prime}$ and, therefore, also $E^{\prime \prime}$ fall off so rapidly far from the charge-current distribution that they do not contribute to the radiation field, for which the electromagnetic fields fall off like $1 / r$. Hence we can calculate the radiation field from $\mathrm{D}^{\prime}$ alone.

Inowing $\mathrm{D}_{\omega}^{\prime \prime}(\mathbf{r})$, the right-hand side of (2.12) becomes known, and this equation can be treated as an equation for $\mathbf{D}_{\omega}{ }^{\prime}(\mathbf{r})$. We introduce the quantity $\mathbf{g}(\mathbf{r}, t)$ such that

$$
\mathbf{g}_{\omega}(\mathbf{r})=\mathbf{j}_{\omega}(\mathbf{r})+i\left(c^{2} / 4 \pi \omega\right) \text { curl curl } \mathbf{E}_{\omega}^{\prime \prime}(\mathbf{r})-i(\omega / 4 \pi) \mathbf{D}_{\omega}^{\prime \prime}(\mathbf{r}) .
$$

This quantity, which has the dimensions of a current, is presumed known. Although the $\mathrm{g}$ field can easily be shown to be transverse, it is not the transverse part of the $\mathbf{j}$ field. In terms of $\mathbf{g}_{\omega},(2.12)$ can be written

$$
\text { curl curl } \mathbf{E}_{\omega}{ }^{\prime}-\left(\omega^{2} / c^{2}\right) \mathbf{D}_{\omega}^{\prime}=i\left(4 \pi \omega / c^{2}\right) \mathbf{g}_{\omega} .
$$

In the next section we shall show how this equation can be solved for $\mathbf{D}_{\omega}^{\prime}$ in terms of $\mathbf{g}_{\omega}$ and, hence, in terms of $\mathbf{j}_{\omega}$.

\section{SOLUTION OF THE EQUATION FOR D}

We shall adopt the notation that the space-time Fourier transform of a field $f(\mathbf{r}, t)$ is $f_{\omega, q}$, given by

$$
\int_{\omega, \mathbf{q}}=\frac{1}{(2 \pi)^{4}} \int d \mathbf{r} \int_{-\infty}^{\infty} d l e^{-i(\mathbf{q} \cdot \mathbf{r}-\omega t)} f(\mathbf{r}, t)=\frac{1}{(2 \pi)^{3}} \int d \mathbf{r} e^{-i \mathbf{q} \cdot \mathbf{r}} f_{\omega}(\mathbf{r}) .
$$

The inverse transform is given by

$$
f(\mathbf{r}, t)=\int d \mathbf{q} \int_{-\infty}^{\infty} d \omega e^{i(\mathbf{q} \cdot \mathbf{r}-\omega t)} f_{\omega, \mathbf{q}} \quad f_{\omega}(\mathbf{r})=\int d \mathbf{q} e^{i \mathbf{q} \cdot \mathbf{r}} f_{\omega \cdot \mathbf{q}} .
$$

If now we take the space Fourier transform of both sides of (2.19) and use (2.3) we obtain:

$$
\begin{aligned}
\left(1-n^{2} \eta_{1}\right) \mathbf{D}_{\omega, \mathrm{q}}^{\prime}-n^{2}\left(\eta_{3}-\eta_{2}\right) \hat{b} \cdot \mathbf{D}_{\omega, \mathrm{q}}^{\prime}(\hat{b}-\hat{k} \cdot \hat{b} \hat{k})-i n^{2} \eta_{2} \hat{k} \cdot \hat{b} \hat{k} \times \mathbf{D}_{\omega, \mathrm{q}}^{\prime} \\
=-(4 \pi i / \omega) \mathbf{g}_{\omega, \mathrm{q}}
\end{aligned}
$$

where we have set

$$
\mathrm{q}=n(\omega / c) \hat{k}
$$

6 The total charge is, of course, independent of time. 
with $\hat{k}$ a unit vector pointing in the direction of $\mathrm{q}$. In obtaining (3.3) we have used the fact that, since $\mathbf{D}_{\omega}{ }^{\prime}(\mathbf{r})$ is a transverse field,

$$
\hat{k} \cdot \mathbf{D}_{\omega \cdot q}^{\prime}=0 .
$$

Equation (3.3) is an inhomogeneous linear equation for $\mathbf{D}_{\omega, \mathbf{q}}^{\prime}$. It may be readily verified that the solution which vanishes when $\mathbf{g}_{\omega, \mathbf{q}}$ vanishes, i.e., when there is no source for the field, is:

$$
\mathbf{D}_{\omega, \mathbf{q}}^{\prime}=-(4 \pi i / \omega \Delta) \mathbf{J}_{\omega, \mathbf{q}}
$$

where

$$
\mathrm{J}_{\omega, \mathbf{q}}=\left(1-n^{2} \eta_{1}\right) \mathbf{g}_{\omega, \mathbf{q}}-n^{2}\left(\eta_{3}-\eta_{1}\right)\left(\hat{k} \times \hat{b} \cdot \mathbf{g}_{\omega, \mathbf{q}}\right) \hat{k} \times \hat{b}+i n^{2}{ }_{r_{2}} \hat{k} \cdot \hat{b} \hat{k} \times \mathbf{g}_{\omega, \mathbf{q}} \text {, }
$$

and where

$$
\Delta=\left(1-n^{2} \eta_{1}\right)^{2}-\left(1-n^{2} \eta_{1}\right)\left(\eta_{3}-\eta_{1}\right) n^{2} \sin ^{2} \theta-n^{4} \eta_{2}{ }^{2} \cos ^{2} \theta
$$

in which $\theta$ is the angle between $\hat{k}$ and $\hat{b}$, i.e.,

$$
\hat{k} \cdot \hat{b}=\cos \theta .
$$

Note that $\Delta$ can be written in the form:

$$
\Delta=\left[1-\left(n^{2} / n_{1}^{2}\right)\right]\left[1-\left(n^{2} / n_{2}^{2}\right)\right],
$$

where $n_{1}(\omega, \theta)$ and $n_{2}(\omega, \theta)$ are the indices of refraction for the two modes of propagation of plane waves of frequeney $\omega$ and with wave normal making an angle $\theta$ with the external magnetic field. ${ }^{7}$ Using the expressions (2.5) one can show:

$$
n_{1.2}^{2}=1-\frac{x}{1-\frac{1}{2} \frac{y^{2}}{1-x} \pm \sin ^{2} \theta}+\sqrt{\frac{1}{4(1-x)^{2}}+y^{2} \cos ^{2} \theta} .
$$

Consider now the quantity $\mathrm{J}_{\omega, q}$, which we would like to express in terms of $\mathrm{j}_{\omega, \mathrm{q}}$, the transform of the given current density. If we take the lourier transform of (2.18) we obtain:

$$
\mathbf{g}_{\omega, \mathbf{Y}}=\mathbf{j}_{\omega, \mathbf{q}}-i(\omega / 4 \pi) \mathbf{D}_{\omega, \mathbf{q}}^{\prime \prime}-i(\omega / 4 \pi) n^{2} \hat{k} \times\left(\dot{k} \times \mathbf{E}_{\omega, \mathbf{q}}^{\prime \prime}\right),
$$

where $\mathbf{E}_{\omega, q}^{\prime \prime}$ is given by $(2.3)$ when $\mathbf{D}_{\omega}$ is replaced by $\mathbf{D}_{\omega, q}^{\prime \prime}$. We can express $D_{\omega . q}^{\prime \prime}$ in terms of $\mathbf{j}_{\omega, q}$ by taking the transform of (2.13) and (2.9) and then eliminating $\rho_{\omega, q}$, giving

$$
\mathbf{q} \cdot \mathbf{D}_{\omega, \mathbf{q}}^{\prime \prime}=-(4 \pi i / \omega) \mathbf{q} \cdot \mathbf{j}_{\omega, \mathbf{q}} .
$$

7 See Ref. 1. A more succinct discussion of the propagation characteristics is given by Ford (6). 
But $\mathbf{D}_{\omega}^{\prime \prime}(\mathbf{r})$ is a longitudinal field, which implies that

$$
\mathrm{D}_{\omega, \mathrm{q}}^{\prime \prime}=\hat{k} \cdot \mathbf{D}_{\omega, \mathrm{q}}^{\prime \prime} \hat{k}
$$

and, together with (3.13), that

$$
\mathrm{D}_{\omega, \mathrm{q}}^{\prime \prime}=-(4 \pi i / \omega) \hat{k} \cdot \mathbf{j}_{\omega, \mathrm{q}} \dot{k} \equiv-(4 \pi i / \omega) \mathbf{j}_{\omega, \mathrm{q}}^{\prime \prime}
$$

where $\mathbf{j}_{\omega, q}^{\prime \prime}$ is the transform of the longitudinal part of the $\mathbf{j}$ field. Using (3.15), the expression (3.12) for $\mathbf{g}_{\omega, q}$ becomes:

$$
\mathbf{g}_{\omega, \mathbf{q}}=\mathbf{j}_{\omega, \mathbf{q}}^{\prime}+n^{2} \hat{k} \cdot \mathbf{j}_{\omega, \mathbf{q}}^{\prime \prime}\left[\left(\eta_{; s}-\eta_{1}\right) \hat{b} \cdot \hat{k}(\hat{b}-\hat{k} \cdot \hat{b} \hat{k})-i \eta_{2} \hat{k} \times \hat{b}\right],
$$

where $j_{\omega, q}^{\prime}$ is the transform of the transverse part of the $\mathbf{j}$ field. That is,

$$
\mathbf{j}_{\omega, \mathbf{q}}^{\prime} \equiv \mathbf{j}_{\omega, \mathbf{q}}-\mathbf{j}_{\omega, \mathbf{q}}^{\prime \prime} \equiv \mathbf{j}_{\omega, \mathbf{q}}-\hat{k} \cdot \mathbf{j}_{\omega, \mathbf{q}} \hat{k}
$$

Finally, inserting (3.16) in the expression (3.7) for $\mathrm{J}_{\boldsymbol{\omega}, \mathbf{q}}$, we find

$$
\begin{aligned}
& \mathrm{J}_{\omega, \mathrm{q}}=\left(1-n^{2} \eta_{1}\right) \mathbf{j}_{\omega, \mathrm{q}}^{\prime}-n^{2}\left(\eta_{3}-\eta_{1}\right)\left(\hat{k} \times \hat{b} \cdot \mathbf{j}_{\omega, \mathrm{q}}^{\prime}\right) \hat{k} \times \hat{b} \\
&+i n^{2} \eta_{2} \hat{k} \cdot \hat{b} \hat{k} \times \mathbf{j}_{\omega . \mathrm{q}}^{\prime}+n^{2} \hat{k} \cdot \mathbf{j}_{\omega . \mathrm{q}}^{\prime \prime}\left\{\left[\left(1-n^{2} \eta_{1}\right)\left(\eta_{3}-\eta_{1}\right)-n^{2}{\eta_{12}}^{2} \mid \hat{k}\right.\right. \\
&\left.\quad \cdot \hat{b}(\hat{b}-\hat{k} \cdot \hat{b} \hat{k})-i \eta_{2}\left(1-n^{2} \eta_{3}\right) \hat{k} \times \hat{b}\right\},
\end{aligned}
$$

which is the desired expression for $J_{\omega, q}$ in terms of $\mathbf{j}_{\omega, q}$.

One may readily verify that the relation (3.18) is equivalent to the following relation between the corresponding time Fourier transformed quantities $\mathbf{J}_{\omega}(\mathbf{r})$ and $\mathbf{j}_{\omega}(\mathbf{r})$.

$$
\begin{aligned}
\mathrm{J}_{\omega}(\mathbf{r})=(1 & \left.+\eta_{1} \frac{c^{2}}{\omega^{2}} \boldsymbol{\nabla}^{2}\right) \mathbf{j}_{\omega}{ }^{\prime}+\left(\eta_{3}-\eta_{1}\right) \frac{c^{2}}{\omega^{2}}(\hat{b} \times \boldsymbol{\nabla})\left(\hat{b} \cdot \boldsymbol{\nabla} \times \mathbf{j}_{\omega^{\prime}}\right) \\
& -i \eta_{2} \frac{c^{2}}{\omega^{2}}(\hat{b} \cdot \boldsymbol{\nabla})\left(\boldsymbol{\nabla} \times \mathbf{j}_{\omega}{ }^{\prime}\right)-\frac{c^{2}}{\omega^{2}}\left\{\left[\left(1+\eta_{1} \frac{c^{2}}{\omega^{2}} \nabla^{2}\right)-\eta_{2}{ }^{2} \frac{c^{2}}{\omega^{2}} \boldsymbol{\nabla}^{2}\right]\right. \\
& \left.\left.\left.\cdot(\hat{b} \cdot \boldsymbol{\nabla})\left(\boldsymbol{\nabla} \times\left(\hat{b} \times \mathbf{j}_{\omega}{ }^{\prime \prime}\right)\right)+i \eta_{2}\left(1+\eta_{3} \frac{c^{2}}{\omega^{2}} \boldsymbol{\nabla}^{2}\right)\right) \hat{b} \times \boldsymbol{\nabla}\right) \boldsymbol{\nabla} \cdot \mathbf{j}_{\omega}{ }^{\prime \prime}\right\} .
\end{aligned}
$$

This expression, while quite complicated, is of the form of a linear differential operator acting on the transverse and longitudinal parts of the time transform of the current density. It does not, however, follow that $J_{\omega}(r)$ vanishes for those regions of space where $\mathbf{j}_{\omega}(r)$ vanishes, i.e., outside the region to which the chargecurrent distribution is confined. The point is that $\mathbf{j}_{\omega}{ }^{\prime}(\mathbf{r})$ and $\mathbf{j}_{\omega}{ }^{\prime \prime}(\mathbf{r})$ do not separately vanish outside the charge-current distribution, only their sum vanishes. Indeed, an explicit expression for $\mathbf{j}_{\omega}{ }^{\prime \prime}(\mathbf{r})$ is given by

$$
\mathbf{j}_{\omega}^{\prime \prime}(\mathbf{r})=-(1 / 4 \pi) \operatorname{grad} \int d \mathbf{r}^{\prime}\left[\operatorname{div} \mathbf{j}_{\omega}\left(\mathbf{r}^{\prime}\right) /\left|\mathbf{r}-\mathbf{r}^{\prime}\right|\right],
$$


which clearly need not vanish for points $\mathbf{r}$ outside the charge-current distribution. However, for points far from the charge-current distribution one does see from (3.20) that:

$$
\begin{aligned}
\mathbf{j}_{\omega}^{\prime \prime}(\mathbf{r}) \sim-(1 / 4 \pi) \operatorname{grad}\{(1 / r) & \int d \mathbf{r}^{\prime} \operatorname{div} \mathbf{j}_{\omega}\left(\mathbf{r}^{\prime}\right) \\
& \left.+\left(1 / r^{3}\right) \mathbf{r} \cdot \int d \mathbf{r}^{\prime} \mathbf{r}^{\prime} \operatorname{div} \mathbf{j}_{\omega}\left(\mathbf{r}^{\prime}\right)+\cdots\right\} .
\end{aligned}
$$

The first term in this expansion vanishes, being the integral of the divergence of a field which vanishes outside a finite region, and, hence $j_{\omega}{ }^{\prime \prime}(r)$ falls off for large $r$ at least as fast as $1 / r^{3}$. Since, outside the charge-current distribution, $\mathbf{j}_{\omega}{ }^{\prime}(\mathbf{r})$ must be equal in magnitude, but opposite in sign, to $\mathbf{j}_{\omega}{ }^{\prime \prime}(\mathbf{r})$, it follows by inspection of the expression (3.19) that far from the charge-current distribution $\mathrm{J}_{\omega}(\mathbf{r})$ falls off at least as fast as $1 / r^{3}$.

We return now to the problem of determining $\mathbf{D}_{\omega}{ }^{\prime}(\mathbf{r})$. Forming the inverse transform of (3.6) and using (3.10) we have:

$$
\mathbf{D}_{\omega}^{\prime}(\mathbf{r})=-(4 \pi i / \omega) \int d \mathbf{q} e^{i \mathbf{q} \cdot \mathbf{r}}\left[n_{1}{ }^{2} n_{2}{ }^{2} /\left(n^{2}-n_{1}{ }^{2}\right)\left(n^{2}-n_{2}{ }^{2}\right)\right] \mathbf{J}_{\omega, \mathbf{q}},
$$

But

$$
\mathrm{J}_{\omega, \mathbf{q}}=\left[1 /(2 \pi)^{3}\right] \int d \mathbf{r}^{\prime} e^{-i \mathbf{q} \cdot \mathbf{r}^{\prime}} \mathrm{J}_{\omega}\left(\mathbf{r}^{\prime}\right)
$$

so that, exchanging the order of integration, we may write:

$$
\mathbf{D}_{\omega}^{\prime}(\mathbf{r})=-(4 \pi i / \omega) \int d \mathbf{r}^{\prime} K\left(\mathbf{r}-\mathbf{r}^{\prime}\right) \mathrm{J}_{\omega}\left(\mathbf{r}^{\prime}\right)
$$

where

$$
K(\mathbf{r})=\left[1 /(2 \pi)^{3}\right] \int d \mathbf{q} e^{i \mathbf{q} \cdot \mathbf{r}}\left[n_{1}{ }^{2} n_{2}{ }^{2} /\left(n^{2}-n_{1}{ }^{2}\right)\left(n^{2}-n_{2}{ }^{2}\right)\right]
$$

In this last expression we should perhaps remind ourselves that $n$ is defined by (3.4), while $n_{1}$ and $n_{2}$ are given by (3.11) and are functions of the direction of q. If we introduce spherical coordinates in the $q$ integration, then $K(\mathbf{r})$ can be written:

$$
K(\mathbf{r})=\dot{(} \omega / 2 \pi c)^{3} \int d \Omega \int_{0}^{\infty} d n e^{i n(\omega / c) \hat{k} \cdot \mathbf{r}}\left[n^{2} n_{1}^{2} n_{2}^{2} /\left(n^{2}-n_{1}^{2}\right)\left(n^{2}-n_{2}^{2}\right)\right],
$$

${ }^{8}$ The asymptotic form for large $r$ of Fourier integrals of this general form is discussed by Lighthill $(7)$. See especially his theorem $2($ p. 414$)$ of which our result (3.35) is a special case. 
where $\mathrm{d} \Omega$ is the e'ement of solid angle in the integral over the directions of $\grave{k}$. We now observe that if we replace $k$ by $-k$ and $n$ by $-n$ the integrand of (3.26) is unchanged while

$$
\int d \Omega \int_{0}^{\infty} d n \rightarrow \int d \Omega \int_{-\infty}^{j} d n
$$

Hence, if we average the resulting expression with $(3.25)$ we obtain the expression:

$$
K(\mathbf{r})=\frac{1}{2}(\omega / 2 \pi c)^{3} \int d \Omega \int_{-\infty}^{\infty} d n e^{i n(\omega / c) \hat{k} \cdot \mathbf{r}}\left[n^{2} n_{1}{ }^{2} n_{2}{ }^{2} /\left(n^{2}-n_{1}{ }^{2}\right)\left(n^{2}-n_{2}{ }^{2}\right)\right] .
$$

The integral over $n$ may now be performed by closing the path of integration with a semicircle at infinity in the upper or lower half of the complex $n$-plane, depending upon whether $\omega \dot{k} \cdot \mathbf{r}$ is positive or negative. The integral along the semicircle vanishes because of the exponential factor and, therefore, the result of the integration is, from Cauchy's theorem, $2 \pi i$ times the sum of the residues at the poles enclosed within the contour. Hence, after some rearrangement (3.28) becomes:

$$
K(\mathbf{r})=i \pi(\omega / 2 \pi c)^{2} \int d \Omega\left[n_{1}{ }^{2} n_{2}{ }^{2} /\left(n_{1}{ }^{2}-n_{2}{ }^{2}\right)\right]\left[n_{1} e^{i n_{1}(\omega / c) \hat{k} \cdot \mathbf{r}}-n_{2} e^{i n_{2}(\omega / c) \hat{k} \cdot \mathbf{r}}\right] .
$$

In this expression $n_{1}$ and $n_{2}$ are to be chosen as those roots of the expressions (3.11) whose imaginary part is positive, and the range of the angular integration is restricted to those values of $k$ for which $\omega \hat{k} \cdot \mathbf{r}$ is positive, i.e., to a hemisphere.

The remaining angular integration in (3.29) cannot be done in closed form in general. However, we are primarily interested in the fields in the radiation zone far from the charge-current distribution and, therefore, in the asymptotic value of $K(\mathbf{r})$ for large $\mathbf{r}$. This value can be obtained from (3.29) by the method of stationary phasc. For simplicity we consider the ease where the collision frequency $\nu$, is much smaller than all the other frequencies in the problem and may be set equal to zero. The expression (3.11) for $n_{1}{ }^{2}$ and $n_{2}{ }^{2}$ is then real and only two possibilities arise: (1) If $n_{1}{ }^{2}$ (or $n_{2}{ }^{2}$ ) is negative then $n_{1}$ is imaginary; the corresponding plane wave mode of propagation is an exponentially damped, nonpropagating mode. For such values of $n_{1}$ the contribution to the integral (3.29) will be exponentially small for large $r$ and may therefore be neglected. (2) If $n_{1}{ }^{2}$ (or $n_{2}{ }^{2}$ ) is positive then $n_{1}$ is real, the corresponding plane wave mode is a propagating mode. Here, while the integrand is not small for large $r$, in the course of the integration the exponential factor will oscillate rapidly and will tend to cancel except in the neighborhood of those values of $\hat{k}$ where the quantity $n_{1} k \cdot \mathbf{r}$ is stationary. To find these stationary points we write

$$
\hat{k}=\left(\hat{k}_{0}+\varepsilon\right) / \sqrt{1+\epsilon^{2}}, \quad \varepsilon \cdot \hat{k}_{0}=0
$$


and expand in power of $\epsilon=|\varepsilon|$ :

$$
\begin{aligned}
n_{1} \hat{k} \cdot \mathbf{r}=\left[n_{1} \hat{k} \cdot \mathbf{r}\right]_{0}+\left[n_{1} \mathbf{r} \cdot \boldsymbol{\varepsilon}+n_{1}^{\prime} \hat{k} \cdot \mathbf{r} \hat{b} \cdot \boldsymbol{\varepsilon}\right]_{0}-1 / 2\left[n_{1} \hat{k} \cdot \mathbf{r} \epsilon^{2}+n_{1}^{\prime} \dot{k} \cdot \mathbf{r} \hat{k} \cdot \hat{b} \epsilon^{2}\right. \\
\left.-2 n_{1}{ }^{\prime} \hat{b} \cdot \varepsilon \mathbf{r} \cdot \mathbf{\varepsilon}-n_{1}{ }^{\prime \prime} \dot{k} \cdot \mathbf{r}(\hat{b} \cdot \mathbf{\varepsilon})^{2}\right]_{0}+\cdots .
\end{aligned}
$$

Here the square brackets are all to be evaluated at $\dot{k}=\hat{k}_{0}$ and the prime denotes differentiation with respect to $\cos \theta=k \cdot \hat{b}$. The stationary points of the integrand in (3.29) are those directions $k_{0}$ for which the first-order terms in (3.31) vanish. Since $\hat{k}_{0} \cdot \varepsilon=0$, we see that this implies that

$$
k_{0}=\frac{n_{1} \mathbf{r}+n_{1}^{\prime} \hat{k}_{0} \cdot \mathbf{r} \hat{b}}{k_{0} \cdot \mathbf{r}\left(n_{1}+n_{1}^{\prime} \hat{k}_{0} \cdot \hat{b}\right)},
$$

which is an implicit equation for $k_{0}$. Evaluating the slowly varying parts of the integrand at $k_{0}$, we find that the contribution to (3.29) from the neighborhood of such a stationary point is:

$$
\begin{aligned}
K(\mathbf{r}) \sim i \pi\left(\frac{\omega}{2 \pi c}\right)^{3}\left\{\left[\frac{n_{1}^{3} n_{2}^{2}}{n_{1}{ }^{2}-n_{2}{ }^{2}}\right]_{0} e^{i(\omega / \boldsymbol{c})\left[n_{1} \hat{k} \cdot \mathrm{r}\right]_{\|}} \int d \boldsymbol{\varepsilon}\right. \\
\left.\cdot \exp \left(-i \frac{\omega r}{2 c} \sum_{i=1}^{2} \sum_{j=1}^{2} A_{i j} \epsilon_{i} \epsilon_{j}\right)+1 \rightleftarrows 2\right\},
\end{aligned}
$$

where

$\sum_{i=1}^{2} \sum_{j=1}^{2} A_{i j} \boldsymbol{\epsilon}_{i} \boldsymbol{\epsilon}_{j}=\frac{1}{r}\left[n_{1} \hat{k} \cdot \mathbf{r} \epsilon^{2}+n_{1}^{\prime} \hat{k} \cdot \mathbf{r} \hat{k} \cdot \hat{b} \epsilon^{2}-2 n_{1}{ }^{\prime} \hat{b} \cdot \boldsymbol{\varepsilon} \mathbf{r} \cdot \boldsymbol{\varepsilon}-n_{1}^{\prime \prime} \hat{k} \cdot \mathbf{r}(\hat{b} \cdot \boldsymbol{\varepsilon})^{2}\right]_{0}$.

In these expressions the symbol $1 \rightleftarrows 2$ denotes the result of interchanging the subscripts 1 and 2 in the first term in the curly bracket. In the remaining integral in (3.33) we may extend the range of integration over all $\varepsilon$ perpendicular to $k_{0}$. This is then a two-dimensional Gaussian integral and the result of the integration is

$$
K(\mathbf{r}) \sim \frac{\pi}{r}\left(\frac{\omega}{2 \pi c}\right)^{2}\left\{\left[\frac{n_{1}^{3} n_{2}^{2}}{n_{1}^{2}-n_{2}^{2}}\right]_{0}\left(\operatorname{det} A_{i j}\right)^{-1 / 2} e^{i(\omega / c)\left[n_{1} \hat{k} \cdot \mathbf{r}\right]_{0}}+1 \rightleftarrows 2\right\} .
$$

Here det $A_{i j}$ denotes the determinant of the $2 \times 2$ matrix $A_{i j}$. Using (3.35), which defines $A_{i j}$, and the expression (3.32) for $\hat{k}_{0}$ we find:

$$
\begin{aligned}
\left(\operatorname{det} A_{i j}\right)=\left[\frac { n _ { 1 } ( n _ { 1 } + n _ { 1 } ^ { \prime } \operatorname { c o s } \theta ) } { n _ { 1 } ^ { 2 } + n _ { 1 } ^ { \prime 2 } \operatorname { s i n } ^ { 2 } \theta } \left(n_{1}^{2}+\right.\right. & n_{1} n_{1}^{\prime} \cos \theta \\
& \left.\left.+2 n_{1}^{\prime 2} \sin ^{2} \theta-n_{1} n_{1}^{\prime \prime} \sin ^{2} \theta\right)\right]_{10} .
\end{aligned}
$$

In obtaining this expression it has been found convenient to rearrange (3.32) so that it takes the form of an explicit expression for $\mathbf{r}$ in terms of $\hat{k}$ : 


$$
\mathbf{r} / r=\left[n_{1} \hat{k}_{0}-n_{1}^{\prime}\left(\hat{b}-\hat{k}_{0} \cdot \hat{b} \hat{k}_{0}\right)\right] / \sqrt{n_{1}{ }^{2}+n_{1}^{\prime 2} \sin ^{2} \theta}
$$

It may be readily verified that this relation is equivalent to (3.32).

Returning now to the expression (3.24) for $\mathbf{D}_{\omega}{ }^{\prime}(\mathbf{r})$, we note that, since $\mathbf{J}_{\omega}(\mathbf{r})$ falls off like $1 / r^{3}$ for large $r$, the asymptotic form of $\mathbf{D}_{\omega}{ }^{\prime}(\mathbf{r})$ for large $r$ is obtained by replacing $K\left(\mathbf{r}-\mathbf{r}^{\prime}\right)$ in the integrand by its asymptotic form given by (3.3.5). We find

$\mathbf{D}_{\omega}^{\prime}(\mathbf{r}) \sim-\frac{i \omega}{r c^{2}}\left\{\left[\frac{n_{1}{ }^{3} n_{2}{ }^{2}}{n_{1}{ }^{2}-n_{2}{ }^{2}}\right]_{0}\right.$

$$
\left.\cdot\left(\operatorname{det} A_{i j}\right)^{-1 / 2} e^{i \mathbf{q}_{1} \cdot \mathbf{r}} \int d \mathbf{r}^{\prime} e^{-i \mathbf{q}_{1} \cdot \mathbf{r}^{\prime}} \mathrm{J}_{\omega}\left(\mathbf{r}^{\prime}\right)+1 \rightleftarrows 2\right\} \text {, }
$$

where

$$
\mathbf{q}_{1}=(\omega / c)\left[n_{1} \hat{k}\right]_{0} .
$$

The remaining integral is just the space Fourier transform of $\mathbf{J}_{\omega}\left(\mathbf{r}^{\prime}\right)$, and, hence,

$$
\mathbf{D}_{\omega}{ }^{\prime}(\mathbf{r}) \sim-\frac{i \omega(2 \pi)^{3}}{r c^{2}}\left\{\left[\frac{n_{1}^{3} n_{2}{ }^{2}}{n_{1^{2}}-n_{2}{ }^{2}}\right]_{0}\left(\operatorname{det} A_{i j}\right)^{-1 / 2} e^{i \mathbf{q}_{1} \cdot \mathbf{r}} \mathbf{J}_{\omega \cdot \mathbf{q}_{1}}+1 \rightleftarrows 2\right\} .
$$

This is the solution of our problem, that of determining the far field form of $\mathbf{D}_{\omega}{ }^{\prime}(\mathbf{r})$. In this expression $n_{1}{ }^{2}$ and $n_{2}{ }^{2}$ arc given by (3.11), (det $\left.A_{i j}\right)$ by $(3.36)$, $\mathrm{J}_{\omega, \mathbf{q}}$ by (3.18), and $\mathbf{q}_{1}$ by (3.39). The unit vector $\dot{k}_{0}$ which appears in all of these expression is given implicitly by ( 3.37 ). Finally we should recall that $n_{1}$ must be chosen as that square root of $n_{1}^{2}$ for which $i q_{1} \cdot r$ has a negative real part.

\section{THE LOW-FREQUENCY LIMIT}

Although (3.40) is an explicit solution to the problem of finding the asymptotic fields it is very complicated in form and hence difficult to discuss. Since the most striking effects of the anisotropy introduced by the external magnetic field occur at frequencies below the plasma frequency $\omega_{p}$ and the electron gyrofrequency $\omega_{11}$, we shall consider the limit of frequencies $\omega \ll \omega_{0}, \omega_{p}$ in which ease the results of Section III become simpler in form.

In this limit it can be readily shown from (3.11) that the expressions for the indices of refraction of the two modes of plane wave propagation become:

$$
n_{1}^{2}=\omega_{p}^{2} / \omega \omega_{0} \cos \theta, \quad n_{2}{ }^{2}=-\omega_{p}^{2} / \omega \omega_{0} \cos \theta
$$

Here we see that there is one propagating mode, with real index of refraction and one nonpropagating mode, with imaginary index of refraction. If we restrict our discussion to positive frequencies and angles $\theta \leqq \pi / 2$, then $n_{1}{ }^{2}$ is always positive and the corresponding mode is the propagating mode. For this mode the relation (3.37) becomes:

$$
\mathbf{r} / r=[2 \cos \theta \hat{k}+(\hat{b}-\hat{k} \cdot \hat{b} \hat{k})] / \sqrt{ } / 3 \cos ^{2} \theta+1 .
$$


This is an implicit equation from which $\hat{k}$ is to be determined when the direction in which the radiation is to be observed is given. (Remember $\mathbf{r}$ points from the source to the observer.) If we denote the angle between $\mathbf{r}$ and $\hat{b}$ by $\psi$, i.e.,

$$
\cos \psi=\mathbf{r} \cdot \hat{b} / r
$$

then from (4.2) it can be shown that:

$$
\sin \psi=\sin \theta \cos \theta / \sqrt{1+3 \cos ^{2} \theta} .
$$

Inspection of this equation shows us that for all angles $\theta, \sin \psi \leqq 1 / 3$, i.e.,

$$
\psi \leqq \arcsin 1 / 3 \cong 20^{\circ},
$$

with the maximum angle $\psi$ oecurring when $\cos \theta=1 / \sqrt{3}$. In other words the radiation field is confined within the two sheets of a right circular cone, whose vertex is at the source and whose axis is along the direction of the external magnetic field. Note also that for every $\psi$ within this cone there are two angles $\theta$ fulfilling (4.4), one greater the other less than $\operatorname{arcos} 1 / \sqrt{3}$.

Using the expression (4.1) for $n_{1}{ }^{2}$, the expression (3.36) for ( $\operatorname{det} A_{i j}$ ) becomes:

$$
\left(\operatorname{det} A_{i j}\right)=n_{1}^{2}\left(3 \cos ^{2} \theta-1\right) / 2\left(3 \cos ^{2} \theta+1\right) .
$$

The expression (3.18) for $\mathrm{J}_{\omega, \mathbf{q}}$ is evaluated using (4.1) and the low-frequency limits of the quantities $\eta_{1}, \eta_{2}$, and $\eta_{3}$ given in $(2.5)$. We find:

$$
\mathbf{J}_{\omega, \mathbf{q}}=\mathbf{j}_{\omega, \mathbf{q}}^{\prime}+i \hat{k} \times \mathbf{j}_{\omega, \mathbf{q}}^{\prime}-\left(\hat{k} \cdot \mathbf{j}_{\omega, \mathbf{q}}^{\prime \prime} / \cos \theta\right)[i \hat{k} \times \hat{b}+(\hat{b}-\hat{k} \cdot \hat{b} \hat{k})] .
$$

Putting these results together the expression (3.40) becomes

$$
\mathbf{D}_{\omega}{ }^{\prime}(\mathbf{r}) \sim \frac{i \omega n_{1}{ }^{2}(2 \pi)^{3}}{r c^{2}} \sqrt{\frac{3 \cos ^{2} \theta+1}{2\left(3 \cos ^{2} \theta-1\right)}} e^{i \mathbf{q}_{1} \cdot \mathbf{r}} \mathrm{J}_{\omega, \mathbf{q} 1} .
$$

Here we see that the field diverges when $\theta=\operatorname{arcos} 1 / \sqrt{3} \cdot{ }^{9}$ This is just the angle corresponding to points on the cone within which the radiation field is confined. Although the fields diverge on this cone, there is no physical difficulty since, as we shall see explicitly in the example in the next section, the total radiated field energy remains finite. The additional result of interest which we see in (4.8) is that the intensity of radiation from a purely longitudinal current is comparable with that from a transverse current.

\section{RADIATION FROM A RADIAL CHARGE BURST}

As an application of the general results of this paper we consider in this section the problem of determining the low-frequency radiation from a thin spherical shell of charge which is expanding outward with radial velocity $v$. For $t>0$, the

${ }^{9}$ If the electron collision frequency $\nu$ is kept finite the fields do not actually diverge on the cone but become very large. 
current density will be:

$$
\mathbf{j}(\mathbf{r}, \mathrm{t})=(Q v / 4 \pi)\left(\mathbf{r} / \mathbf{r}^{3}\right) \delta(r-v t),
$$

where $Q$ is the total charge and $\delta$ is the Dirac delta function. This is a purely longitudinal current which will not radiate in an isotropic medium but which will radiate in a plasma in the presence of an external magnetic field.

The first step is the calculation of the space-time Fourier transform of the current density (5.1).

$$
\begin{aligned}
\mathbf{j}_{\omega \cdot \mathbf{q}} & =\frac{1}{(2 \pi)^{4}} \int d \mathbf{r} \int_{-\infty}^{\infty} d t e^{-i(\mathbf{q} \cdot \mathbf{r}-\omega t)} \mathbf{j}(\mathbf{r}, t) \\
& =\frac{Q}{4 \pi(2 \pi)^{4}} \int d \mathbf{r} \frac{\mathbf{r}}{r^{3}} e^{-i \mathbf{q} \cdot \mathbf{r}} e^{i(\omega r / r)} \\
& =i \hat{k} \frac{Q}{(2 \pi)^{4}} \int_{0}^{\infty} d r e^{i(\omega / \mathbf{r}) r}\left(\frac{\cos q r}{q r}-\frac{\sin q r}{q^{2} r^{2}}\right) .
\end{aligned}
$$

Here we should remind ourselves that $\hat{k}$ is a unit vector in the direction of $q$. If in this last integral we introduce $q r$ as a new variable and write $q v / \omega=n \beta$, where

$$
n=c q / \omega
$$

and

$$
\beta=v / c
$$

we find we can write:

$$
\mathbf{j}_{\omega, \mathbf{q}}-i \hat{k}\left[Q /(2 \pi)^{4} q\right] I(n \beta)
$$

where

$$
I(x)=\int_{0}^{\infty} d u e^{i u / x}\left[(\cos u / u)-\left(\sin u / u^{2}\right)\right] .
$$

It is possible to show that in general

$$
\begin{aligned}
I(x)= & -1+(1 / 2 x) \ln [(1+x) /(1-x)], \quad x<1 \\
& -1+(1 / 2 x) \ln [(x+1) /(x-1)-i \pi / 2 x, \quad x>1
\end{aligned}
$$

However, we shall restrict our discussion to the case $n \beta \gg 1$, i.e., to the case where the radial velocity of the burst is much greater than the phase velocity of the electromagnetic waves. The radiation might then be properly called Cerenkov radiation (see Ref. 2 , esp. Chapter II). It is a simple matter to show that for $n \beta$ large, $I(n \beta)=-1$, and (5.5) becomes:

$$
\mathrm{j}_{\omega, Q}=\frac{-i k Q}{(2 \pi)^{4} q}
$$


Inserting this result in the expression (4.8) for $\mathbf{D}_{\omega}^{\prime}(\mathbf{r})$, valid at low frequencies, we find

$$
\mathrm{D}_{\omega}^{\prime}(\mathbf{r}) \sim-\frac{Q n_{1}}{2 \pi r c} \sqrt{\frac{3 \cos ^{2} \theta+1}{2\left(3 \cos ^{2} \theta-1\right)}} e^{i \mathbf{q}_{1} \cdot \mathbf{r}} \frac{1}{\cos \theta}[(\hat{b}-\hat{k} \cdot \hat{b} k)+i \hat{k} \times \hat{b}] .
$$

The asymptotic electric field, $\mathbf{E}_{\omega}{ }^{\prime}(\mathbf{r})$, is obtained using $(2.3)$, which in the lowfrequency limit becomes:

$$
\mathbf{E}_{\omega}{ }^{\prime}(\mathbf{r})=\left(i / n_{1}{ }^{2} \cos \theta\right) \hat{b} \times \mathbf{D}_{\omega}^{\prime}(\mathbf{r})
$$

where we have used the low-frequency limit expression (4.1) for $n_{1}{ }^{2}$. Inserting (5.9) gives:

$$
\begin{aligned}
& \mathbf{E}_{\omega}{ }^{\prime}(\mathbf{r}) \sim-{ }_{2 \pi r c n_{1}}^{Q} \sqrt{\frac{3 \cos ^{2} \theta+1}{2\left(3 \cos ^{2} \theta-1\right)}} \frac{e^{i \mathbf{q}_{1} \cdot \mathbf{r}}}{\cos \theta} \\
& {\left[(\hat{b}-\hat{k} \cdot \hat{b} \hat{k})+i \hat{k} \times \hat{b}-\frac{\sin ^{2} \theta}{\cos \theta} \hat{k}\right] .}
\end{aligned}
$$

Finally, we obtain $\mathbf{B}_{\omega}(\mathbf{r})$ from (2.7),

$$
\mathbf{B}_{\omega}(\mathbf{r}) \sim-\frac{Q}{2 \pi r c} \sqrt{\frac{3 \cos ^{2} \theta+1}{2\left(3 \cos ^{2} \theta-1\right)}} \frac{e^{i \mathbf{q}_{1} \cdot \mathbf{r}}}{\cos \theta}[\hat{k} \times \hat{b}-i(\hat{b}-\hat{k} \cdot \hat{b} \hat{k})] .
$$

The complex Poynting vector, $\mathrm{S}_{\omega}$, is defined as (see, e.g., Ref. 5, pp. 135-37)

$$
\mathbf{S}_{\omega}=(c / 2) \mathbf{E}_{\omega} \times \mathbf{B}_{\omega}^{*} .
$$

The real part of this vector is equal to the energy flux density per frequency interval. If $P(\omega ; \psi, \Phi) d \Omega d \omega$ is the total energy with frequency between $\omega$ and $\omega+d \omega$ radiated into solid angle $d \Omega=\sin \psi d \psi d \Phi$, then

$$
P(\omega ; \psi, \Phi)=2 \lim _{r \rightarrow \infty} r \mathbf{r} \cdot R l\left\{\mathbf{S}_{\omega}\right\}
$$

where the factor 2 accounts for the redundancy of positive and negative frequencies. Here $\psi$ and $\Phi$ are, respectively, the polar and azimuthal angle of the position vector $r$, which points from the source to the field point. If we choose the direction of the external magnetic field as polar axis, then $\psi$ is related to $\theta$ by (4.4). Using (5.11) and (5.12) we find that

$$
R l\left\{\mathbf{S}_{\omega}\right\}=\frac{Q^{2}}{16 \pi^{2} n_{1} c} \frac{\left(3 \cos ^{2} \theta+1\right)^{3 / 2} \sin ^{2} \theta \mathbf{r}}{\left|3 \cos ^{2} \theta-1\right| \cos ^{3} \theta} \frac{r^{3}}{3}
$$

where the expression (4.2) for $r / r$ has been used. Note that this result says that the radiated energy propagates radially away from the source, as one must expect. Inserting in $(5.14)$ we find

$$
P(\omega ; \psi, \Phi)=\frac{Q^{2}}{8 \pi^{2} n_{1} c} \frac{\left(3 \cos ^{2} \theta+1\right)^{3 / 2} \sin ^{2} \theta}{\left|3 \cos ^{2} \theta-1\right| \cos ^{3} \theta} .
$$


The total radiated energy per frequency interval is obtained by integrating over all solid angles within the cone to which the radiation is confined.

$$
\begin{aligned}
P(\omega) & =\int d \Omega P(\omega ; \psi, \Phi) \\
& =\frac{Q^{2}}{4 \pi c} \int d \psi \sin \psi \frac{\left(3 \cos ^{2} \theta+1\right)^{3 / 2} \sin ^{2} \theta}{\left|3 \cos ^{2} \theta-1\right| \cos ^{3} \theta n_{1}}
\end{aligned}
$$

In the integrand the angles $\psi$ and $\theta$ are related by (4.4) from which one can readily show that

$$
\sin \psi d \psi=\frac{3 \cos ^{2} \theta-1}{\left(3 \cos ^{2} \theta+1\right)^{3 / 2}} \cos \theta \sin \theta d \theta .
$$

Hence, if the variable of integration is changed from $\psi$ to $\theta(5.17)$ becomes:

$$
P(\omega)=\frac{Q^{2}}{4 \pi c} \sqrt{\frac{\omega \omega_{0}}{\omega_{p}^{2}}} \int_{0}^{\pi} d \dot{\lambda} \frac{\sin ^{3} \theta}{|\cos |^{3 / 2}},
$$

where the expression (4.1) for $n_{1}^{2}$ has been used. Here we see explicitly that, since the integral does not diverge on the cone bounding the radiation field, i.e., at $\theta=\operatorname{arcos} 1 / \sqrt{3}$, the divergence of the fields on this cone does not lead to any physical difficulty. However the integral in (5.19) does diverge at $\theta=\pi / 2$. It can be shown that this divergence is related to the breakdown of low-frequency approximation at such angles. A more careful analysis shows that the integrand in fact drops sharply to zero for angles such that

$$
|\cos \theta| \lesssim \frac{\omega \sqrt{\omega_{0}^{2}+\omega_{p}^{2}}}{\omega_{p} \omega_{0}}
$$

If we approximate this behavior by simply cutting off the integral for such angles we find:

$$
P(\omega) \cong\left(Q^{2} / \pi c\right)\left[\omega_{0} / \omega_{p}^{1 / 2}\left(\omega_{0}^{2}+\omega_{p}^{2}\right)^{1 / 4}\right]
$$

where we have kept only the largest terms.

From the above discussion we see that the largest contribution to the total radiated energy comes from those values of $\theta$ near $\pi / 2$. From the relation $(4.4)$ between $\psi$ and $\theta$ we see that this corresponds to very small values of $\psi$. In other words, the most intense radiation is confined within a narrow cone, angular width of order $\omega \sqrt{\omega_{0}^{2}+\omega_{p}^{2}} / \omega_{p} \omega_{0}$, about the direction of the cxternal magnetic field. From (5.11) we see that the electric field in this wave is longitudinal, i.e., it points in the direction of the wave normal $k$, which means that the electric: field oscillates in a direction perpendicular to the direction of propagation. On the other hand from (5.9) and (5.12) we see that the dielectric displacement and the magnetic field of the wave are transverse. In fact these two fields rotate with uniform angular velocity in a plane perpendicular to the wave normal, a plane 
which includes the direction of propagation. The peculiar form of this electromagnetic wave reflects the extreme anisotropy of the medium.

Although the current (5.1) is undoubtedly only the crudest sort of an approximation to the current arising from a nuclear explosion in the upper atmosphere, the general features of the low-frequency radiation should still follow. There should be a very narrow intense burst of electromagnetic radiation along the earth's magnetic field lines. Since both the plasma frequency and the electron gyrofrequency in the ionosphere are of the order of a megacycle per sec, ${ }^{10}$ the spectrum of this radiation is confined to frequencies of the order of 10 to $100 \mathrm{kc}$. There is a difficulty, however, in that one would expect that this radiation would be totally reflected at the lower boundary of the ionosphere, since the wave normal is nearly parallel to the boundary and the index of refraction changes from a very large value in the ionosphere to a value of order unity in the atmosphere. On the other hand one would expect in general that a narrow bundle of radiation which is totally reflected at an interface between two media will give rise to surface waves propagating along the interface away from the region of impact. "In the case of the lower boundary of the ionosphere whese waves would surely be coupled to surface waves on the earth, which could be detected.

Receiven: May 1, 1961

\section{REFERENCEN}

1. J. A. Ratcliffe, "The Magneto-Ionic Theory and Its Applications to the Ionosphere." Cambridge Univ. Press, London and New York, 1959.

2. J. V. Jelley, “Cerenkov Radiation,” pp. 23-24. Pergamon Press, New York, 1958.

3. D. Bu rnetr, Proc. Cambridge Phil. Soc. 27, 578 (1931).

4. W. K. H. Panofsky and M. Phillips, "Classical Electricity and Magnetism," pp. 1-6. Addison-Wesley, Reading, Massachusetts, 1955.

5. J. A. Stratton, "Electromagnetic Theory," p. 166. Mc(Graw-Hill, New York, 1951.

6. G. W. Ford, "Electrodynamics in Low Density Plasmas. I." Technical Report 60-P00102184, Space Technology Laboratories, Ine., Los Angeles, July, 1960.

7. M. J. Lighthili, Trans. Roy. Soc. A252, 397 (1960).

3. G. P. Kiriper, "The Atmospheres of the Earth and Planets," pp. 8, 9. Univ. of Chicago Press, Chicago, 1952.

9. J. Picht, Ann. Physik [5] 3, 433 (1929).

10 The plasma frequency $\omega_{p}$ varies with height through the variation of electron density $N$ with height. If $N=10^{5} \mathrm{~cm}^{-3}$ then $\omega_{1} / 2 \pi \cong 3 \times 10^{6} \mathrm{sec}^{-1}$. This value of $N$ corresponds to a height of about $200 \mathrm{~km}$. See Ref. 8 .

11 For the case isotropic media the finite pulse effect was first studied by Picht $(9)$. 